\title{
Metástasis en iris de un carcinoma de mama: ¿modifica el tratamiento la historia natural de la enfermedad?
}

\author{
J. A. PÉREZ-FIDALGO, J. VILA ARTEAGA, I. CHIRIVELLA GONZÁLEZ, \\ R. MARTÍNEZ BELDA, B. BERMEJO DE LASHERAS, S. ROSELLÓ KERANËN, \\ A. FONS MORENO, A. LLUCH HERNÁNDEZ \\ Servicio de Oncología y Oftalmología. Hospital Clínico Universitario. Valencia
}

BREAST CANCER METASTASES IN THE IRIS: DOES TREATMENT MODIFIES NATURAL HISTORY OF THE ILLNESS?

\begin{abstract}
RESUMEN
El 4-5\% de las pacientes con cáncer de mama presentan metástasis oculares. Presentamos a una mujer de 30 años con un carcinoma ductal infiltrante de mama pT2N2M0 HER2 positivo. A los seis meses de finalizar el tratamiento presentó una recaída sistémica con múltiples localizaciones metástaticas por lo que inició tratamientos combinados de trastuzumab mantenido y quimioterapia. Tras 4 años con trastuzumab de mantenimiento presenta en iris de ojo derecho multiples micronódulos blanquecinos. Solo un 3-7.8\% de las metástasis oculares se localizan en el iris. Con la terapia mantenida con trastuzumab la historia natural de la enfermedad avanzada ha cambiado, diversos estudios analizaron si existe mayor frecuencia de metástasis cerebrales en pacientes tratados con trastuzumab que en la era pretrastuzumab. La infrecuente aparición de metástasis en úvea anterior hace difícil establecer si es un hecho espontáneo o si está favorecido por el tratamiento con trastuzumab.
\end{abstract}

PALABRAS CLAVE: Carcinoma de mama. Metástasis oculares. Iris. Trastuzumab.

\section{ABSTRACT}

The 4-5\% of the breast cancer patients have metastases in the eye. We present the case of a 30-year-old woman with an infiltrant duct carcinoma of the breast pT2N2MO HER2 positive. Six months after primary radical treatment she had a systemic relapse with multiples metastatic sites, so several treatment with trastuzumab in combination with chemotherapy were started. After 4 years patient presented multiple white-coloured micronodules in the iris of the right eye. Only a 3-7.8\% of ocular metastases are located in the iris. With mantenaince therapy with trastuzumab natural history of the illness has changed. Several studies had analyzed if metastases in the brain during treatment with trastuzumab have increased in comparison with the pretrastuzumab era. The infrequent presentation of metastases in the anterior uveal makes difficult to establish if it is an spontaneous fact or if it is favoured by trastuzumab treatment.

KEY WORDS: Breast cancer. Ocular metastases. Iris. Trastuzumab.

Pérez-Fidalgo JA, Vila Arteaga J, Chirivella González I, Martínez Belda R, Bermejo de Lasheras B, Roselló Keranën S, Fons Moreno A, Lluch Hernández. A. Metástasis en iris de un carcinoma de mama: ¿modifica el tratamiento la historia natural de la enfermedad? An Med Interna (Madrid) 2008; 25: 178-180.

\section{INTRODUCCIÓN}

La lesión maligna ocular más frecuente son las metástasis. El tumor primario que más frecuentemente metastatiza en el ojo es el cáncer de mama (1).

Aproximadamente entre el 6-9\% de las pacientes con cáncer de mama presentan metástasis al diagnóstico (2), siendo pulmón, hígado y hueso los órganos más frecuentemente afectados.

Aún así, las metástasis oculares de cáncer de mama al diagnóstico o en recaída son muy infrecuentes estimándose entre un $4-5 \%$ de las pacientes (3).

El polo posterior del ojo es la localización más frecuente de metástasis uveales, en una serie de 520 metástasis oculares (4) hasta un $88 \%$ eran coroideas mientras que la afecta- ción de iris y cámara anterior fue mucho más infrecuente $(11 \%)$.

Las metástasis oculares son difusas, multifocales, hipopigmentadas y con frecuencia bilaterales. En la mayoría de los casos son diagnosticadas sin necesidad de biopsia, considerándose que con examen clínico con lámpara de hendidura y ecografía las tasas de precisión diagnóstica rondan el $99 \%(5)$.

\section{CASO APORTADO}

El caso que presentamos a continuación trata de una mujer de 30 años que acudía en Abril del 2001 por un nódulo en mama izquierda 
que tras ser biopsiado se diagnosticó de un carcinoma ductal. El estudio de extensión incluyendo rastreo óseo, ecografía abdominal y radiografía de tórax fue normal.

A la exploración física se palpaba un nódulo de 6 x $7 \mathrm{~cm}$ y 2 adenopatías de 3 y $4 \mathrm{~cm}$ de diámetro. Con el diagnóstico de carcinoma ductal de mama izquierda cT4b N1 inició tratamiento con quimioterapia neoadyuvante dentro de ensayo clinico con paclitaxel, adriamicina liposomal y trastuzumab del que recibió 5 ciclos con toxicidad dermatológica y cardiaca que motivó suspensión del $6^{\circ}$ ciclo.

Se realizó entonces una tumorectomía con linfadenectomía reglada detectándose en la pieza quirúrgica un carcinoma ductal infiltrante grado histológico III de 2,5 x 1,5 x 1,2 cm con afectación de 3 de los 17 ganglios aislados, receptores hormonales negativos, erbB2 positivo $+++/+++$.

Con el diagnóstico de carcinoma ductal infiltrante pT2 N2(3/17) M0 con erbB2 positivo se decidió continuar tratamiento adyuvante con paclitaxel y trastuzumab del cual recibió 8 ciclos. Posteriormente recibió radioterapia adyuvante y herceptin semanal de mantenimiento con buena tolerancia.

A los seis meses se detecta una recaída sistémica ósea, hepática, ganglionar y pulmonar.

Se decide entonces inicio de quimioterapia paliativa con docetaxel y trastuzumab cada 21 días del que recibió 7 ciclos y tras objetivarse una respuesta parcial se mantuvo tratamiento con trastuzumab semanal.

En julio de 2003 se detecta progresión de enfermedad por lo que se administra segunda línea de quimioterapia paliativa con vinorelbina y trastuzumab semanal, tratamiento que mantiene durante once meses hasta que en junio de 2004 se aprecia una progresión de enfermedad a nivel cerebral.

La paciente recibe radioterapia holocraneal y se inicia posteriormente tratamiento con quimioterapia paliativa de tercera línea con gemzitabina y trastuzumab.

Tras 5 meses de tratamiento se objetiva una nueva progresión de enfermedad que motiva cuarta línea de quimioterapia paliativa esquema paclitaxel, carboplatino y trastuzumab junto con ácido zoledrónico mensual. Tras haber administrado 4 ciclos la paciente refiere aparición de una alteración de la agudeza visual con visión borrosa sin apreciarse ojo rojo.

La exploración oftalmológica demuestra una agudeza visual de 0.6 en ambos ojos. Ala lampara de hendidura se aprecia en ojo derecho a nivel temporal del iris lesiones sobreelevadas a modo de micronódulos de diámetro inferior al milimetro de aspecto gelatinoso y color blanquecina, algunas de las cuales tienden a confluir y muestran en su base neovasos (Fig. 1), las lesiones provocan rigidez iridiana y retraen el borde temporal de la pupila mostrando un ligero ectropion uveal, el fondo de ojo es normal.

Se decidió realización de un TAC toracoabdominal que confirmó progresión de enfermedad sistémica por lo que en un contexto de enfermedad diseminada progresiva y con una exploración altamente sugestiva se consideró que los micronódulos iridianos eran metástasis por carcinoma de mama.

La paciente recibió tratamiento con radioterapia externa consiguiéndose una respuesta a nivel iridiano con la desaparición de la mayor parte de los micronódulos. Esta pendiente de iniciar nueva línea de quimioterapia con trastuzumab.

\section{DISCUSIÓN}

La presentación de metástasis en iris por un carcinoma de mama es muy infrecuente. Tras realizar una búsqueda bibliográfica en pubmed con las palabras clave BREAST CANCER, UVEAL, IRIS, OCULAR y METASTASIS entre los años 1970 y 2005 hemos encontrado sólo 6 casos clínicos de metástasis iridianas de carcinoma de mama (6-11) descritos en la literatura.

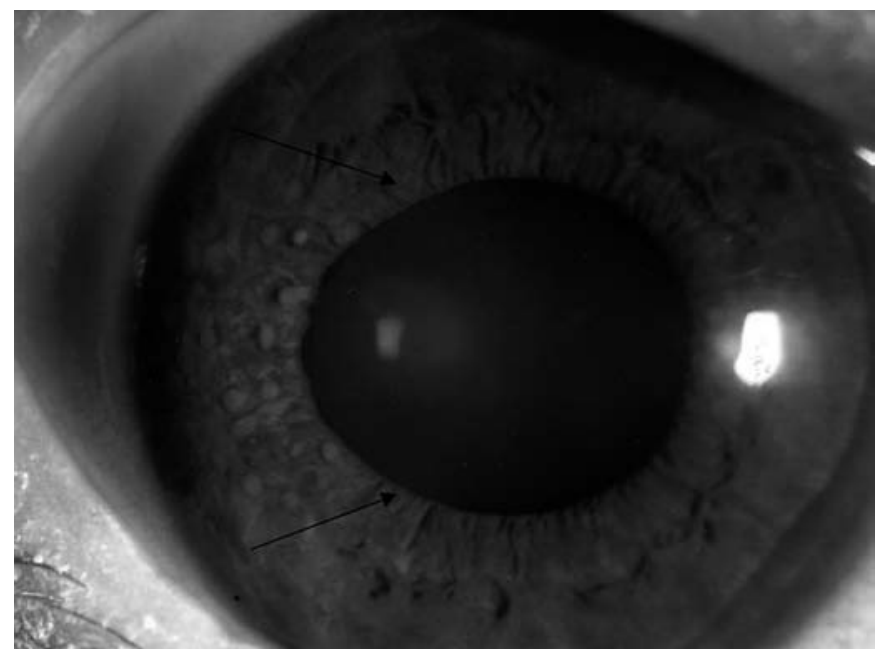

Fig. 1. Micronódulos metastásicos en iris en el área limitada por las flechas.

A pesar de la escasez de casos descritos, en 1995 Shields et al (12) en una revisión de 512 pacientes con metástasis en uvea, identificaron 40 casos $(7.8 \%)$ de metástasis en cámara anterior, de los cuales 16 eran metástasis de un cáncer de mama. Por otro lado Demirci et al (13) en una revisión posterior de 264 pacientes con metástasis en úvea por carcinoma de mama únicamente describe un $3 \%$ (8 pacientes) con afectación del iris. En ambas series el tratamiento empleado fue la radioterapia en ocasiones seguida de quimioterapia sistémica.

Las metástasis en iris pueden presentar un espectro de variaciones clínicas pero son suficientemente específicas como para poder diferenciar la neoplasia intraocular con otras inflamaciones. La presentación clínica más frecuente es en forma de micronódulos difusos e hipopigmentados de color amarilloblanquecina. Con frecuencia son bilaterales. La presencia de un ectropion uveal, en la exploración oftalmológica es un signo muy significativo de la presencia de una lesión neoplásica.

El tratamiento específico para la mayoría de las metástasis oculares es la radioterapia. Es un tratamiento no invasivo y bien tolerado, fundamentalmente en las metástasis coroides. No obstante, dado que raramente las metástasis oculares son únicas, la radioterapia debe de complementarse con tratamiento sistémico para control de la enfermedad a distancia (5).

La infrecuente localización metastásica de esta paciente nos obliga a preguntarnos si se trata de una rareza de su historia natural o si el tratamiento ha podido influir en esta atípica localización de una metástasis. Con frecuencia la afectación metastásica del ojo y del sistema nervioso central (SNC) ocurre de forma simultánea, algunas series identifican que alrededor del $30 \%$ de pacientes con metástasis oculares presenta además afectación del SNC (14). Por ello se aconseja la evaluación cerebral con TAC y resonancia magnética a toda paciente con metástasis oculares. Todo ello parece sugerir una etiopatogenia similar de la afectación metastásica de ambos órganos.

Aunque nuestra paciente no presentaba afectación del SNC, es llamativo que la afectación del iris ocurre en el contexto de un tratamiento prácticamente de forma mantenida con trastuzumab.

Históricamente, en la era pre-trastuzumab, las metástasis en el sistema nervioso central (SNC) eran relativamente poco frecuentes, únicamente un $10 \%$ de las pacientes las presentaba. 
Entonces, la supervivencia media tras la afectación de SNC estaba en torno al año. Sin embargo algunos autores han comenzado a comunicar tasas de recaidas en SNC en pacientes HER-2 positivas tratadas con trastuzumab que rondan el 30$40 \%$, siendo la supervivencia media de unos 2 años (15). Estos resultados inducen a pensar que el trastuzumab ejerce un excelente control de la enfermedad sistémica pero fracasa en la prevención de recaídas en localizaciones como en el SNC.

Varios estudios intentaron aclarar esta observación, así Lai $y$ cols. (16) en un estudio de cohortes retrospectivo compararon el riesgo de presentar metástasis cerebrales entre una cohorte de 264 pacientes que no recibieron trastuzumab frente a otra con 79 pacientes que no recibieron dicho tratamiento. Los autores no encontrando asociación entre la administración de trastuzumab y la aparición de metástasis cerebrales. Sin embargo la validez de este estudio puede ser cuestionada, ya que existían diferencias significativas entre la proporción de pacientes HER 2 + entre ambas cohortes $(47,3 \%$ de HER 2 +en el grupo que recibía trastuzumab frente a un 14,3\% HER2 + en el grupo sin trastuzumab). De hecho, un importante análisis retrospectivo realizado en 9524 pacientes por el International Breast Cancer Group (17), identificó la sobreexpresion de HER2 como uno de los factores predictivos de recaída en SNC, junto con el tamaño tumoral $>2 \mathrm{~cm}$, los receptores de estrógenos negativos, los ganglios positivos, el grado histológico de 3 y la edad inferior a 35 años.

Lower y cols. (18) estudiaron el riesgo de metástasis en SNC de 299 pacientes estratificadas según hubieran recibido o no tratamiento con trastuzumab. Los resultados de este estudio demostraron que el trastuzumab prevenía frente a las metástasis extracerebrales pero no había diferencias en la proporción de metástasis en el SNC (25\% en el grupo sin trastuzumab frente a $31 \%$ en el grupo con trastuzumab) sin embargo la supervivencia de las metástasis en SNC estaba aumentada en el grupo del trastuzumab. Esto sugiere que la terapia ni previe- ne ni aumenta la aparición de metástasis cerebrales, aunque si que previene la recaída sistémica lo que tiene un claro efecto en la supervivencia.

Sin embargo estos estudios tienen la limitación de la estratificación por sobreexpresión de HER2, lo cual hace que el establecimiento de conclusiones sobre si el papel del aumento de las metástasis en SNC es por el trastuzumab o por el HER2+. Por ello, un estudio reciente (19) comparó en 750 pacientes todas ellas HER2+ la proporción de metástasis cerebrales entre un control histórico en la era pre-trastuzumab que no había recibido el fármaco y un segundo grupo en la era post que si lo recibió. Los resultados del estudio confirman, tras un ajuste por otras variables pronosticas que incluían edad, estadio al diagnóstico y grado histológico que las pacientes HER 2 + que recibían trastuzumab tenían 2,84 veces más riesgo que las pacientes HER2 + que no lo recibían.

Estos datos respaldan la hipótesis de que si bien trastuzumab es muy eficaz en el tratamiento de las metástasis óseas, hepáticas y pulmonares parece ineficaz en prevenir las metástasis cerebrales. Este efecto podría deberse a la menor difusión del fármaco a través de barrera hemato-encefálica o a una mayor predilección de las células HER2 + por el sistema nervioso central.

La infrecuente aparición de metástasis en úvea anterior hace difícil establecer si es un hecho espontáneo o si está favorecido por el tratamiento recibido. Sería imposible establecer si la causa de una localización metastásica infrecuente está en la presencia de factores pronósticos adversos, como la edad (nuestra paciente tenía menos de 35 años), la sobreexpresión HER2, el TNM o si bien se debe factores derivados del tratamiento recibido durante una larga enfermedad.

No obstante parece posible que al igual que ocurre en las metástasis cerebrales, el tratamiento con trastuzumab al alargar la evolución de la enfermedad modifique su historia natural aumentando la aparición de localizaciones metastásicas poco habituales.

\section{Bibliografía}

1. Freedman MI, Folk JC. Metastatic tumors to the eye and orbit. Arch Ophtalmol 1987; 71: 373

2. Greenlee R, Hill-Harmon M, Murray T. Cancer statistics 2001. CA Cancer J Clin 2001; 51: 15-36.

3. Kreusel KM, Wiegel T, Stange M, Bornfeld N, Foerster MH. Intraocular metastases of metastatic breast carinoma in the woman. Incidence, risk factors and therapy. Ophtalmologe. 2000; 97: 342-6.

4. Shields Cl, Shields JA, Gross NE, Schwartz GP, Lally SE. Survey of 520 eyes with uveal metastases. Ophtalmology 1997; 104: 1265-75.

5. McCormick BA, Abramson DH. Ocular metastases. En: Harris JR, Lippman ME, Morrow M, Osborne CK, editors. Diseases of the breast. $3^{\text {th }}$ ed. Philadelphia: Lippincott, Williams and Wilkins; 2004. p.1257-60.

6. Martin M, Zierhut D, Piroth M, Gutwein S, Debus J., Dithmar S. Solitary iris metastasis from breast cancer. Effective local therapy with electron beam irradiation. Ophtalmologe 2006; 103: 48-51.

7. Gutierrez Sanchez E, Alonso Alonso I. Metastasis en el iris: un hallazgo casual. Arch Soc Esp Oftalmol 2000; 75: 689-91.

8. Reddy SC, Madhavan M, Mutum SS. Anterior uveal and episcleral metastases from carcinoma of the breast. Ophtalmologica 2000; 214: 368-72.

9. Hoche A, Blum M, Semeniuk C, Lorenz P, Kosmehl H, Strobel J. Secondary glaucoma caused by iris tumor. Iris metastasis of adenocarcinoma of the breast. Ophtalmologe 1999; 6: 342-3.

10. Escobar R, Ronquillo A, Escobar F, Álvarez-Morujo M. Trabeculectomía por glaucoma secundario a metástasis de carcinoma de mama. Rev Med Univ Navarra 1989; 33: 131-2

11. Bonnet JL, de Marigny G, Marthouret M. Metastasis in the iris of a can- cer of the breast. Bull Soc Ophtalmol Fr 1970; 70: 283-5.

12. Shields JA, Shields CL, Kiratli H, de Potter P. Metastatic tumors to the iris in 40 patients. Am J Ophtalmol 1995; 119: 422-30.

13. Demirci H, Shields CL, Chao AN, Shields JA. Uveal metastasis from breast cancer in 264 patients. Am J Ophtalmol 2003; 136: 264-71.

14. Ratanatharathorn V, Powers W, Grimm J. Eye metastasis from carcinoma of the breast: Diagnosis, radiation treatment and results. Cancer Treat Rev 1991; 18: 261

15. Dawson SJ, Ranieri NF, Snyder RD, McLahlan SA, Burns WI, Newnham GM, Francis PA, Dowling AJ. Trastuzumab and CNS metastases in women with HER-2 positive metastatic breast cancer. J Clin Oncol 2005 ASCO Annual Meeting Proceedings 2005; 23 (16 Supl.): 683.

16. Lai R, Dang CT, Malkin MG, Abrey LE. The risk of central nervous system metastases after trastuzumab therapy in patients with breast carcinoma. Cancer 2004; 104 (4): 810-16.

17. Pestalozzi BC, Zahrieh D, Price KN, Holmberg SB, Lindtner J, Collins J, et al. For the International Breast Cancer Study Group. Identifying breast cancer patients at risk for central nervous system metastases in trials of the International Breast Cancer Study Group. Ann Oncol 2006; 17:935-44.

18. Lower EE, Drosick DR, Blau R, Brennan L, Danneman W, Hawley Dk. Increased rate of brain metastases with trastuzumab therapy not associated with impaired survival. Clin Breast Cancer 2003; 4: 114-9.

19. Pinder MC, Chang H, Broglic KR, Michaud LB, Theriault RL, Sahin A, et al. Trastuzumab treatment and the risk of central nervous system metastases. J Clin Oncol 2007 ASCO Annual Meeting Proceedings Part I 2007; 25 (18 Supl.): 1018 